\title{
ON BOUNDED ELEMENTS OF LINEAR ALGEBRAIC GROUPS
}

\author{
BY \\ KWAN-YUK CLAIRE SIT(1)
}

\begin{abstract}
Let $F$ be a local field of characteristic zero and $G$ a connected algebraic group defined over $F$. Let $G$ be the locally compact group of $F$-rational points. One characterizes the group $B(G)$ of $g \in G$ whose conjugacy class is relatively compact. For instance, if $\mathbf{G}$ is $F$-split or reductive without anisotropic factors then $B(G)$ is the center of $G$. If $H$ is a closed subgroup of $G$ such that $G / H$ has finite volume, then the centralizer of $H$ in $G$ is contained in $B(G)$. If, moreover, $H$ is the centralizer of some $x \in G$ then $G / H$ is compact.
\end{abstract}

1. Introduction. For a locally compact group $G$, Tits [12] has described the subgroup $B(G)$ of all elements in $G$ which have precompact conjugacy classes by introducing the concept of automorphism of bounded displacement (a.b.d.). An automorphism $\alpha$ of $G$ is an a.b.d. if the set $\left\{\alpha(g) g^{-1} \mid g \in G\right\}$ has compact closure. It is easy to see that $x \in B(G)$ if and only if the inner automorphism $\alpha_{x}$ of $G$ induced by $x$ is an a.b.d.

Let $F$ be a locally compact nondiscrete field of characteristic zero, $\mathbf{G}$ an algebraic group defined over $F$ and $G$ the $F$-rational subgroup of $\mathbf{G}$. Thus the group $G$ is canonically equipped with a topology of a locally compact group. For an automorphism $\alpha$ of $\mathbf{G}$ defined over $F$, we say that $\alpha$ is an a.b.d. if the restriction $\alpha_{\mid G}$ is an a.b.d. of $G$. In $\S 2$ we study the a.b.d of $\mathbf{G}$ in the spirit of Tits and obtain a structure theorem for $B(G)$. The main results are the following:

2.1. THEOREM. Let $\mathbf{G}$ be a connected algebraic group defined over $F, G$ its $F$-rational subgroup, $\alpha$ an automorphism of $\mathbf{G}$ defined over $F$ and $\mathbf{N}$ the unipotent radical of $\mathbf{G}$.

(i) If $\alpha$ is an a.b.d. of G, then for any unipotent element $n$ of $G, \alpha(n)=n$ and for any torus $\mathbf{T}$ of $\mathbf{G}$ defined and split over $F, \alpha(t)=t$ for all $t \in \mathbf{T}$.

(ii) If for any $n \in N, \alpha(n)=n$ and if for any torus $\mathbf{T}$ of $\mathbf{G}$ defined and split

Received by the editors April 3, 1974.

AMS (MOS) subject classifications (1970). Primary 20G25, 22E20.

Key words and phrases. Local fields, automorphisms of bounded displacement, bounded elements, homogeneous spaces of finite volume, density theorem.

(1) This forms part of the author's doctoral thesis at the Graduate School, City University of New York. 
over $F, \alpha(t)=t$ for all $t \in T$, then $\alpha$ is an a.b.d. (Here $N$ and $T$ are the F-rational points of $\mathrm{N}$ and $\mathrm{T}$ respectively.)

2.2. Corollary. Let $\mathbf{G}$ and $G$ be as in 2.1. Then

$$
B(G)=\bigcap\left\{Z_{G}(T) \mid \mathbf{T} \text { is a torus of } \mathbf{G} \text { defined and split over } F\right\} \cap Z_{G}(N)
$$

where $Z_{G}(T)$ (resp. $Z_{G}(N)$ ) denotes the centralizer of $T$ (resp. $\left.N\right)$ in $G$; in particular, $B(G)$ is closed.

2.3. Corollary. Let $\mathbf{G}$ and $G$ be as in 2.1. If $\mathbf{G}$ is reductive, then $B(G)=$ $\bigcap\{P \mid \mathbf{P}$ is a minimal parabolic $F$-subgroup of $\mathbf{G}\}$.

2.4. Theorem. Let $\mathbf{G}$ and $G$ be as in 2.1. Suppose (i) that $\mathbf{G}$ is F-split or (ii) that $\mathbf{G}$ is reductive with no $F$-anisotropic simple factors. Then there is no nontrivial a.b.d. In particular, $B(G)$ equals the center $Z(G)$ of $G$.

In $\S 2$, we also prove that complex analytic linear groups or simply connected complex analytic groups have no nontrivial complex analytic a.b.d. and so for such groups, $B(G)=Z(G)$ (see 2.8 ).

2.1 and 2.2 are the analogue of Tits' results for real analytic groups while 2.3 is another characterization of $B(G)$ for reductive linear groups. 2.4 and the result for complex analytic groups are analogous to various results of Tits [12] and Greenleaf-Moskowitz-Rothschild [4].

Using techniques suggested in [4] and [10] we prove in $\$ 3$ results for algebraic groups similar to those for semisimple analytic groups [10] and connected locally compact groups [4]. The main theorem is

3.1. THEOREM. Let $\mathbf{G}$ and $G$ be as in 2.1 , then any finite central measure on $G$ is supported on $B(G)$.

A closed subgroup $H$ of a locally compact group $G$ is called a generalized uniform subgroup of $G$ if the homogeneous space $G / H$ of left cosets admits a finite invariant measure. In $\S 4$ we prove results on the centralizer $Z_{G}(H)$ of $H$ in $G$ and a compactness condition of $G / H$ for linear groups all of which have an analogue [4], [5] for connected Lie groups.

4.1. TheOREM. Let $\mathbf{G}, G$ be as in 2.1 and $H$ a generalized uniform subgroup of $G$. Then $Z_{G}(H) \subset B(G)$.

4.2. Corollary. Let $\mathbf{G}, G$ and $H$ be as in 4.1. Then the commutator subgroup of $Z_{G}(H)$ has compact closure.

In [11] an example of a totally disconnected group $G$ such that the commutator subgroup of $Z_{G}(H)$ does not have compact closure is given. In the case 
that $F$ is a $p$-adic field, 4.2 proves the result for a class of totally disconnected groups.

As an analogue of Borel's density theorem [1], we prove

4.3. Corollary. Let G, $G$ and $H$ be as in 4.1. Suppose that $B(G)=$ $Z(G)$, then $Z_{G}(H)=Z(G)$. In particular, this is the case when $\mathbf{G}$ is $F$-split or when $\mathbf{G}$ is reductive with no F-anisotropic simple factors.

4.4. Theorem. Let $\mathbf{G}, G$ and $H$ be as in 4.1. Suppose that $H$ is the centralizer of some element $x \in G$, then $G / H$ is compact.

When $F=\mathbf{R}, 4.4$ is a particular case of a theorem of Mostow [9, Theorem 7.1] and when $F$ is $p$-adic, 4.4 is the analogue of Tamagawa's result [13, Theorem 1].

Finally, I would like to express my gratitude and appreciation to Professors H. Jacquet and M. Moskowitz who gave much encouragement and generous help throughout the writing of this paper.

2. The structure of $B(G)$. Throughout this paper, $F$ denotes a locally compact nondiscrete field of characteristic zero, i.e. $F$ is either the field $\mathbf{R}$ of real numbers, the field $\mathbf{C}$ of complex numbers or a $p$-adic field (i.e. a finite extension of the field $\left.\mathbf{Q}_{p}\right)$. By an $F$-group we mean an algebraic subgroup $\mathbf{G}$ of $\mathrm{GL}(n)$ defined over $F$ where GL( $n)$ denotes the group of $n \times n$ invertible matrices over an algebraically closed extension of the field $F$. By the unipotent radical of $\mathbf{G}$ we mean the maximal (Zariski) connected normal unipotent subgroup $\mathbf{N}$ of $\mathbf{G}$. It is well known that $\mathbf{N}$ is defined and split (triangularizable) over $F$. By an $F$-split torus in $\mathbf{G}$ we mean an algebraic subgroup $\mathbf{T}$ of $\mathbf{G}$ defined and diagonalizable over $F$. An $F$-group $\mathbf{G}$ is said to be $F$-split or split over $F$ if $\mathbf{G}$ has a maximal torus which is $F$-split. We denote by $G$ (resp. $N, T$ ) the subgroup of all $F$-rational points in $\mathbf{G}$ (resp. $\mathbf{N}, \mathbf{T}$ ); in other words $G=\mathbf{G} \cap \mathrm{GL}(n, F)$. It is known that $G$ is Zariskidense in $\mathbf{G}$. By a Borel subgroup of $\mathbf{G}$ we mean a maximal solvable connected subgroup $\mathbf{L}$ of $\mathbf{G}$. Any closed subgroup of $\mathbf{G}$ which contains a Borel subgroup is called a parabolic subgroup. For the general theory of algebraic groups, the reader is referred to [2] and [3]. Recall that an automorphism $\alpha$ of $\mathbf{G}$ defined over $F$ is an a.b.d. of $\mathbf{G}$ if the restriction $\alpha_{\mid G}$ of $G$ is an a.b.d. of $G$, i.e. $\left\{\alpha(g) g^{-1} \mid g \in G\right\}$ has compact closure. An element $x \in G$ is bounded if the inner automorphism $\alpha_{\boldsymbol{x}}$ of $\mathbf{G}$ is an a.b.d. and $B(G)$ is the set of bounded elements.

$\S 2$ concerns itself with the proof of results stated in 2.1, 2.2, 2.3 and 2.4. We begin our proof with the following lemma:

2.5. Lemma. Suppose $X, Y \in M(n, F)$ are nilpotent matrices such that the set $\{(\exp t X)(\exp -t Y) \mid t \in F\}$ has compact closure. Then $X=Y$. 
Proof. Since $X$ and $Y$ are nilpotent, $P(t)=(\exp t X)(\exp -t Y)$ is a polynomial in $t$ with coefficients in $M(n, F)$. In other words each entry $P_{i j}(t)$ of $P(t)$ is a polynomial in $t$ with coefficients in $F$. That the set $\{P(t) \mid t \in F\}$ has compact closure is equivalent to that each set $\left\{\left|P_{i j}(t)\right| \mid t \in F\right\}$ is bounded. Hence each $P_{i j}(t)$ is a constant and $P(t)$ is a constant matrix. Thus $d P / d t=0$ and in particular

$$
0=\left.\frac{d}{d t} P(t)\right|_{t=0}=-Y+X \text {. }
$$

This proves the lemma.

We recall some facts about a torus. The group of all characters of a torus $\mathbf{T}$ defined and split over $F$ is the set of all group homomorphisms $\psi: T \rightarrow$ GL(1). Since $\mathbf{T}$ is $F$-split, all the characters of $\mathbf{T}$ are defined over $F$. Now $\mathbf{T}$ is isomorphic to GL(1) ${ }^{r}$ for some natural number $r$, so we may write for each $t \in \mathrm{T}, t=$ $\left(a_{1}, \ldots, a_{r}\right), a_{i} \in \mathrm{GL}(1)$ and take the $F$-rational points $T$ of $\mathbf{T}$ to be $\mathrm{GL}(1, F)^{r}$. The mappings $\sigma_{i}: \mathrm{T} \rightarrow \mathrm{GL}(1)$ defined by $\sigma_{i}(t)=a_{i}, t \in \mathrm{T}, i=1, \ldots, r$, form a basis for the group of characters of $\mathrm{T}$. Therefore for each character $\psi$ of $\mathrm{T}$,

$$
\psi(t)=\prod_{i=1}^{r} \sigma_{i}(t)^{m(i)}=\prod_{i=1}^{r} a_{i}^{m(i)}
$$

for some integers $m(i)$.

We claim that if the set $\{\psi(t) \mid t \in T\}$ is bounded (with respect to the topology of $F$ ), then $\psi(t)=1$ for all $t \in \mathrm{T}$. Indeed, for each $i=1, \ldots, r$, let $t=$ $\left(1, \ldots, 1, a_{i}, 1, \ldots, 1\right), a_{i} \in F^{\times}$, then we have $\psi(t)=a_{i}^{m(i)}$ and $\left\{d_{i}^{m(i)} \mid a_{i} \in F^{\times}\right\}$ bounded. Therefore, for each $i=1, \ldots, r, m(i)=0$ or equivalently $\psi=1$.

Proof OF 2.1. Let $T$ be the collection of all $F$-split tori $\mathbf{T}$ in $\mathbf{G}$. If $\alpha$ is an $F$-rational a.b.d. of $\mathbf{G}$ and $\mathrm{T} \in T$, then $\alpha(\mathrm{T})$ is again an $F$-split torus. Let $\left\{v_{1}, \ldots, v_{n}\right\}$ be a basis of $F^{n}$ consisting of simultaneous eigenvectors of $\alpha(\mathbf{T})$ and $w=\sum_{i=1}^{n} b_{i} v_{i}$ be a simultaneous eigenvector of $\mathbf{T}$. We have for each $s \in \alpha(\mathbf{T})$, $s\left(v_{i}\right)=\psi_{i}(s) v_{i}, i=1, \ldots, n$, and for each $t \in \mathrm{T}, t(w)=\phi(t) w$ where each $\psi_{i}$, $i=1, \ldots, n$ (resp. $\phi$ ) is a character of $\alpha(\mathrm{T})$ (resp. T).

Since $\alpha$ is an a.b.d., the set

$$
\left\{\alpha(t) t^{-1} \mid t \in T\right\}
$$

has compact closure and hence so does the set $\left\{\alpha(t) t^{-1}(w) \mid t \in T\right\}$ of transforms of $w$ by elements of (1). Now for any $t \in T$, we have

$$
\alpha(t) t^{-1}(w)=\phi\left(t^{-1}\right) \alpha(t)\left(\sum b_{i} v_{i}\right)=\sum b_{i} \phi\left(t^{-1}\right) \psi_{i}(\alpha(t)) v_{i} .
$$

Denote by $\phi^{-1}$ the map defined by $\phi^{-1}(t)=\phi\left(t^{-1}\right)$. It is easy to see that for each $i=1, \ldots, n, \phi^{-1} \cdot\left(\psi_{i} \circ \alpha\right)$ is a character of $\mathrm{T}$ and that for $b_{i} \neq 0$, $\phi^{-1} \cdot\left(\psi_{i} \circ \alpha\right)$ is bounded on $T$. Hence for $b_{i} \neq 0$, we have $\phi^{-1} \cdot\left(\psi_{i} \circ \alpha\right)=1$ or 
$\phi(t)=\psi_{i}(\alpha(t))$ for all $t \in \mathrm{T}$. Hence for all $t \in \mathrm{T}$, we have

$$
t(w)=\phi(t) w=\sum b_{i} \psi_{i}(\alpha(t)) v_{i}=\sum_{b_{i}} \alpha(t)\left(v_{i}\right)=\alpha(t)(w) .
$$

Since $w$ is an arbitrary eigenvector for $t$ and both $t$ and $\alpha(t)$ are semisimple, we have $\alpha(t)=t$ for all $t \in \mathrm{T}$.

Now we show that for any unipotent element $g \in G, \alpha(g)=g$. (It follows from this that $\alpha$ is trivial on $\mathbf{N}$ since $\alpha$ is defined over $F$ and $N$ is Zariski-dense in N.) Let $d \alpha$ be the differential of $\alpha$ and $X$ be the nilpotent matrix in $M(n, F)$ such that $\exp X=g$. Then $d \alpha(X)$ is again nilpotent. Since $\alpha$ is an a.b.d., the set

$$
\left\{\alpha(\exp t X)(\exp t X)^{-1} \mid t \in F\right\}=\{(\exp t d \alpha(X))(\exp -t X) \mid t \in F\}
$$

has compact closure. So by 2.5 , we have $d \alpha(X)=X$. Hence $\alpha(\exp X)=$ $\exp d \alpha(X)=\exp X$ or $\alpha(g)=g$. Thus (i) is proved.

To prove (ii) we show first that for any $\mathrm{T} \in T, g \in G,\left[\alpha(g) g^{-1}, t\right]=1$ for all $t \in \mathrm{T}$. Since $g^{-1} \mathrm{~T} g$ is again a torus defined and split over $F$, we have, for all $t \in \mathrm{T}$,

$$
g^{-1} t g=\alpha\left(g^{-1} t g\right)=\alpha(g)^{-1} t \alpha(g) .
$$

And so $\left[\alpha(g) g^{-1}, t\right]=1$.

Now let $\mathbf{G}=\mathbf{D} \cdot \mathbf{N}$ and $G=D \cdot N$ where $\mathrm{D}$ is a Levi $F$-subgroup of $\mathbf{G}$. Let $\mathbf{P}$ be a minimal parabolic subgroup of $\mathbf{D}$ and $\mathbf{M}$ be a Levi $F$-subgroup of $\mathbf{P}$. Then there exists a maximal $F$-split torus $\mathbf{S}$ in $\mathbf{M}$ such that $\mathbf{M}=Z_{\mathrm{D}}(\mathbf{S})$ and $M / S$ is compact (see [3, Corollary 4.16 and Proposition 9.3]). Hence there is a compact set $C$ in $M$ such that $M=C S$. By a theorem of Bruhat and Tits (see, for example, [7]), we have $D=K M K$ where $K$ is a compact subgroup of $D$. Hence $G=K C S K N$ and for any $g$ in $G$, we have $g=k c s k^{\prime} n$ where $k, k^{\prime} \in K, c \in C, s \in S$ and $n \in N$. So

$$
\begin{aligned}
\alpha(g) g^{-1} & =\alpha(k) \alpha(c) \alpha(s) \alpha\left(k^{\prime}\right) \alpha(n) n^{-1} k^{\prime-1} s^{-1} c^{-1} k^{-1} \\
& =\alpha(k) \alpha(c) s \alpha\left(k^{\prime}\right) k^{\prime-1} s^{-1} c^{-1} k^{-1} \\
& =\alpha\left(k c k^{\prime}\right)\left(k c k^{\prime}\right)^{-1},
\end{aligned}
$$

because $\alpha(n)=n$ and $\left[\alpha\left(k^{\prime}\right) k^{\prime-1}, s\right]=1$. Hence the set $\left\{\alpha(g) g^{-1} \mid g \in G\right\}$ has compact closure and the proof of 2.1 is complete.

REMARK. In the above proof, we note that the compactness of $K$ and $C$ in fact implies that the set $\left\{\alpha(g) g^{-1} \mid g \in G\right\}$ is compact and hence closed. In particular, if $x \in B(G)$, then the conjugacy class $\Gamma_{x}$ of $x$ in $G$ is closed. (In general, for any $x \in G, \Gamma_{x}$ is locally closed, but need not be closed.)

Let us prove 2.2:

Recall that $x \in B(G) \Longleftrightarrow \alpha_{x}$ is an a.b.d. of $\mathbf{G}$. It follows from 2.1 that 


$$
B(G)=\bigcap_{T \in \mathcal{T}} Z_{G}(T) \cap Z_{G}(N) .
$$

As $Z_{\mathrm{G}}(\mathrm{N})$ and $Z_{\mathrm{G}}(\mathrm{T}), \mathbf{T} \in T$, are defined over $F$, so is the subgroup

$$
\mathbf{B}=\bigcap_{\mathbf{T} \in \mathfrak{T}} Z_{\mathbf{G}}(\mathbf{T}) \cap Z_{\mathbf{G}}(\mathbf{N})
$$

and $B(G)$ is exactly the $F$-rational points of $\mathbf{B}$; in particular $B(G)$ is closed. Thus we have proved 2.2.

PROOF OF 2.3. Since $G$ is reductive and

$$
B(G)=\bigcap\left\{Z_{G}(T) \mid \mathrm{T} \in T\right\}=\bigcap\left\{Z_{G}(T) \mid \mathrm{T} \text { is a maximal } F \text {-split torus }\right\},
$$

it suffices to show that

$\bigcap\left\{Z_{G}(T) \mid \mathbf{T}\right.$ is a maximal $F$-split torus $\}=\bigcap\{P \mid \mathbf{P}$ is a minimal parabolic $F$-subgroup $\}$.

Now for any maximal $F$-split torus $\mathbf{T}$ of $\mathbf{G}$, there exist two opposite minimal parabolic $F$-subgroups $\mathbf{P}$ and $\mathbf{P}^{\prime}$ of $\mathbf{G}$ such that $Z_{\mathrm{G}}(\mathrm{T})=\mathbf{P} \cap \mathbf{P}^{\prime}$ and $Z_{G}(T)=P \cap P^{\prime}$. So $\bigcap Z_{G}(T) \supset \bigcap P$. On the other hand for any minimal parabolic $F$-subgroup $\mathbf{P}$ of $\mathbf{G}$, there exists a maximal $F$-split torus $\mathbf{T}$ of $\mathbf{G}$ in $\mathbf{P}$ such that $Z_{\mathbf{G}}(\mathbf{T})$ is a Levi subgroup of $\mathbf{P}$. Hence $\cap Z_{G}(T) \subset \bigcap P$ and the proof of 2.3 is complete.

2.6. Corollary. Let $\mathbf{G}$ be a connected F-group and $\mathrm{B}$ be as above. Then $\mathbf{B}$ is a normal F-subgroup of $\mathbf{G}$ with its unipotent radical central and every F-split torus of $\mathbf{B}$ is central in $\mathbf{B}$. If $\mathbf{G}$ is reductive, then $\mathbf{B}$ is also reductive and quasi F-anisotropic (i.e. any F-split torus of B is central in B. See [3] .).

Proof. Since $B(G)$ is normal in $G$ and since $G$ is Zariski-dense in $\mathbf{G}, \mathbf{B}$ is normal in $\mathbf{G}$. The unipotent radical $\mathbf{U}$ of $\mathbf{B}$ is a normal subgroup of $\mathbf{G}$ contained in $\mathbf{N}$ and hence $[\mathbf{U}, \mathbf{B}]=1$. The rest of the corollary is obvious.

REMARK. When B is reductive, $B(G)$ is compactly generated [3, Theorem 13.4]. When $B$ is not reductive, then in the case where $F$ is a $p$-adic field, $B(G)$ is not compactly generated since $F$ and hence the rational points $U$ of $\mathbf{U}$ are not compactly generated; while in the case where $F=\mathbf{R}$ or $\mathbf{C}, B(G)$ is compactly generated since in this case both the rational points of a Levi subgroup of $B(G)$ and $U$ are compactly generated. In fact, $B(G)$ is compactly generated for any connected locally compact group $G$ [5, Proposition 2]. Though $B(G)$ is not necessarily compactly generated for algebraic groups $G$, it is always an $[F D]^{-}$group as shown in the following corollary. (A locally compact group $G$ is an $[F D]^{-}$ group if the commutator subgroup $G^{\prime}$ of $G$ has compact closure.)

2.7. Corollary. Let $\mathbf{G}$ be a connected F-group. Then $B(G)$ is an $[F D]^{-}$ group. 
Proof. Let $\mathbf{B}$ be as above and let $\mathbf{B}=\mathbf{M} \cdot \mathbf{U}$ and $B(G)=M \cdot U$ where $\mathbf{M}$ is a Levi $F$-subgroup of $B$ and $U$ its unipotent radical. By 2.6, $U$ is central in $B(G)$ and hence $B(G)^{\prime}=M^{\prime}$. Thus it suffices to show $M \in[F D]^{-}$. To see this, we observe first that $M$ is compactly generated [3, Theorem 13.4]. As a closed subgroup of $B(G), M$ is an $[F C]^{-}$group. Therefore by a result of Grosser and Moskowitz [6, Theorem 3.20] that the class of compactly generated $[F C]^{-}$ groups coincides with the class of $[F D]^{-}$groups, we have $M$ an $[F D]^{-}$group and this completes the proof of 2.7.

Proof of 2.4. Let $\alpha$ be an a.b.d of $\mathbf{G}$. First we prove the case when $\mathbf{G}$ is $F$-split. Let $\mathbf{T}$ be an $F$-split maximal torus of $\mathbf{G}$ and $\mathbf{L}$ be a Borel subgroup of G that contains $\mathbf{T}$. If we prove that $\alpha(g)=g$ for all $g \in \mathbf{L}$, then by a result of Borel [2, Corollary 11.4, p. 263], we shall have $\alpha(g)=g$ for all $g \in \mathbf{G}$.

Now $\mathbf{L}=\mathbf{T} \cdot \mathbf{L}_{u}$ where $\mathbf{L}_{\boldsymbol{u}}$ is the subgroup of unipotent elements of $\mathbf{L}[2, \mathrm{p}$. 244]. By 2.1, we have $\alpha(g)=g$ for all $g \in \mathbf{T}$ and for all unipotent elements $g \in G$ and in particular for all $g \in L_{u}$. Since $\alpha$ is defined over $F$ and $L_{u}$ is Zariski-dense in $\mathbf{L}_{u}$, it follows that $\alpha(g)=g$ for all $g \in \mathbf{L}_{u}$. Thus $\alpha(g)=g$ for all $g \in \mathbf{L}$ and $\alpha$ is the identity mapping of $\mathbf{G}$.

Now we prove the case when $\mathbf{G}$ is reductive with no $F$-anisotropic simple factors. Let $H$ be the subgroup of $G$ generated by the unipotent elements of $G$ and the elements of $\bigcap\{T \mid \mathrm{T} \in T\}$ and $\bar{H}$ be the Zariski-closure of $H$ in $\mathbf{G}$. Then $\bar{H}$ is a closed $F$-subgroup of $\mathbf{G}$ [2, Proposition 1.3] and $\alpha=$ id on $\bar{H}$. It is obvious that $\bar{H}$ contains every maximal connected $F$-triangularizable subgroup of $\mathbf{G}$. Hence $(\mathbf{G} / \bar{H})_{F}$ is compact [3, Proposition 9.3]. But $\mathbf{G}$ is reductive with no anisotropic simple factors, thus $\mathbf{G}=\bar{H}$ and the result follows.

EXAMPLES. For a connected $F$-split group $\mathbf{G}$, we know by 2.4 that there are no nontrivial $F$-rational a.b.d. of $\mathbf{G}$, but $\mathbf{G}$, may admit nontrivial non- $F$-rational a.b.d. For example, take $G=\mathbf{G}=\mathbf{C}^{\times}$, then $\mathbf{G}$ is a (Zariski) connected algebraic group defined and split over $\mathbf{C}$. The map $\alpha: \mathbf{G} \rightarrow \mathbf{C}^{\times}$defined by $\alpha(z)=\bar{z}$ for all $z \in \mathbf{G}$ is obviously an a.b.d.; but $\alpha$ is not defined over $\mathbf{C}$. However if we regard $\mathbf{C}^{\times}$as an algebraic group over $\mathbf{R}$, then $\alpha$ is $\mathbf{R}$-rational and is an a.b.d. of $\mathbf{C}^{\times}$. (Here $\mathbf{G}$ is not R-split.)

We note that Lemma 2.5 or rather its proof has another interesting corollary in the case of complex analytic groups, namely:

\subsection{Proposition. Suppose that either}

(i) $G$ is a complex analytic linear group, or

(ii) $G$ is a simply connected complex analytic group.

Then any complex analytic a. b.d. of $G$ is trivial. In particular, since inner automorphisms are complex analytic, $B(G)=Z(G)$. 
2.8 is a special case of [4, Theorems 10.5 and 10.6] where the following is proven: If $G$ is a complex analytic group with a faithful finite-dimensional real analytic linear representation, then $G$ has no nontrivial complex analytic a.b.d. However, the present result is proven by considerably simpler means. We note that if a complex analytic group is neither linear nor simply connected, then the conclusion fails. For example, let $H$ be the Heisenberg group

$$
\left\{\left[\begin{array}{lll}
1 & x & z \\
0 & 1 & y \\
0 & 0 & 1
\end{array}\right] \mid x, y, z \in \mathrm{C}\right\} .
$$

For simplicity, we denote by $(x, y, z)$ a typical element of $H$. It is easy to see that $Z(H)=\{(0,0, z) \mid z \in \mathbf{C}\}$. Let $\Gamma=\{(0,0, k+i m) \mid k, m \in \mathbf{Z}\}$ be a discrete central subgroup of $H$ and $G=H / \Gamma$. Simple computation shows that the commutator subgroup $[H, H]=Z(H)$. Hence $[G, G]=Z(H) / \Gamma$ is compact, and so $G$ is an $[F D]^{-}$group. In particular $G$ is an $[F C]^{-}$group and $B(G)=G$. Therefore any inner automorphism of $G$ is an a.b.d. The present proof of (i) and (ii) requires $\alpha$ to be a complex analytic automorphism as does [4, Theorem 10.6]. Simple examples show that the result fails for real analytic automorphisms of a complex analytic group. However, in the case of [4, Theorem 10.5] it is sufficient to consider only real analytic automorphisms.

Proof OF 2.8. Let $G$ be a complex analytic group and $(\$$ be its Lie algebra. Let $\alpha$ be a complex analytic a.b.d. of $G$ and $d \alpha$ be its differential. Since $G$ is generated by a canonical neighborhood $V$ of 1 in $G$ and $\alpha$ is a homomorphism, it suffices to show that $\alpha(g)=g$ for all $g \in V$.

Now for each $g \in V$, there exists an $x \in($ such that $\exp x=g$. For $t \in \mathrm{C}$, let

$$
\beta(t)=\alpha(\exp t x)(\exp t x)^{-1}=(\exp t d \alpha(x))(\exp -t x) .
$$

Since $\alpha$ is an a.b.d., $\{\beta(t) \mid t \in \mathbf{C}\}$ has compact closure.

If $G$ is linear, then each $(i, j)$ th entry $\beta_{i j}(t)$ of $\beta(t)$ is a bounded complex analytic function on the entire plane. Hence by Liouville's theorem $\beta_{i j}$ is a constant. Since $\beta(0)=I$, it follows that $\beta(t)=\left(\beta_{i j}(t)\right)=I$ for all $t \in \mathbf{C}$. Hence for $t=1, \alpha(g)=g$.

Now let $G$ be simply connected. Consider the adjoint representation Ad: $G$ $\rightarrow \mathrm{GL}(())$. The set $\{\operatorname{Ad}(\beta(t)) \mid t \in \mathrm{C}\}$ again has compact closure. So, as in the linear case, each $\operatorname{Ad}(\beta(t))_{i j}$ is a constant and $\operatorname{Ad}(\beta(t))=I$ for all $t \in \mathbf{C}$. Hence $\{\beta(t) \mid t \in \mathbf{C}\} \subset Z(G)$ and it is easy to see that $\{\beta(t)\}$ is a subgroup of the connected component $Z(G)_{0}$ of $Z(G)$. Since $G$ is simply connected, $Z(G)_{0}$ contains no nontrivial compact subgroup [8]. Therefore $\beta(t)=1$ for all $t \in \mathbf{C}$ and in particular $\alpha(g)=g$. 
3. Layerings of $G$. We use the notations of $\S 2$. Let $I(G)$ denote the group of inner automorphisms of $G$.

Recall that a measure $\mu$ on a locally compact group $G$ is said to be central if $\mu$ is $I(G)$-invariant, i.e., if $\mu\left(\alpha_{x} A\right)=\mu(A)$ for all $\alpha_{x} \in I(G)$ and all Borel sets $A$ in $G$. The main object of this section is to prove 3.1 that any finite central measure of $G$ is supported on $B(G)$. First we need the following technical result.

3.2. THEOREM. Let $\mathbf{G}$ be a connected $F$-group and $G$ the subgroup of $F$ rational elements of $\mathbf{G}$. Then there exists a layering of $G$ terminating at $B(G)$, i. e., a finite collection of closed $I(G)$-invariant subsets $G=X_{0} \supset X_{1} \supset \cdots \supset$ $X_{m}=B(G)$ such that for each $x \in X_{j}-X_{j+1}, j=0,1, \ldots, m-1$, there exists a relative neighborhood $V$ of $x$ in $X_{j}$ with infinitely many pairwise disjoint conjugates.

It is easy to see that by means of simple inductive arguments 3.1 follows from 3.2 immediately.

For detailed discussions of 'layerings' one may refer to [4]. The proof of 3.2 here differs from those of [4] and [10] for semisimple analytic groups and connected locally compact groups mainly in the case when $F$ is a $p$-adic field. We show first that there exist layerings of $G$ terminating at $Z_{G}(N)$ and at $\cap Z_{G}(T)$ $(T \in T)$ respectively. Then by means of the following Lemma 3.3 adapted from [4] and the structure of $B(G)$ (see 2.2), we obtain a layering of $G$ terminating at $B(G)$.

3.3. Lemma [4, Lemma 2.2]. Let $C, D$ be two closed $I(G)$-invariant subsets of a locally compact group $G$ such that there are layerings $G=X_{0} \supset \cdots \supset$ $X_{m}=C$ and $G=Y_{0} \supset \cdots \supset Y_{k}=D$. Then there exists a layering of $G$ that terminates with $C \cap D$.

3.4. Lemma. Let $F^{r}$ be equipped with the topology induced by the norm of $F$. Then for each $x \neq 0 \in F^{r}$, there exist an open neighborhood $E$ of $x$ in $F^{r}$ and a sequence of natural numbers $k_{1}<k_{2}<\cdots$ such that $\delta(i) E$ are pairwise disjoint where we set $\delta(i)=p^{k_{i}}$ with $p>1$ any natural number if $F=\mathbf{R}$ or $\mathbf{C}$ and $\delta(i)=1 / p^{k_{i}}$ with $p$ equal to the characteristic of the residue field if $F$ is noneuclidean.

Proof. We denote by $|x|$ the norm of $x \in F^{r}$. Let $x \neq 0 \in F^{r}$, then $|x| \neq 0$. Let $d$ be a real number such that $0<d<|x|$. The set $E=\left\{x+y \mid y \in F^{r}\right.$, $|y|<d\}$ is obviously an open neighborhood of $x$. Now for any $x+y \in E$, we have

$$
0<|x|-d<|x|-|y| \leqslant|x+y| \leqslant|x|+|y|<|x|+d .
$$


Let $k_{1}$ be the smallest natural number such that

$$
|x|+d<p^{k_{1}}(|x|-d)
$$

where $p$ is the characteristic of the residue field if $F$ is noneuclidean and $p>1$ is any natural number if $F=\mathbf{R}$ or $\mathbf{C}$.

For $i \geqslant 2$, we define $k_{i}$ to be the smallest natural number such that

$$
p^{k_{i-1}}(|x|+d)<p^{k_{i}}(|x|-d) .
$$

Thus the $\delta(i) E$ 's are pairwise disjoint since

$$
\begin{aligned}
\sup _{z \in E}|\delta(i-1) z|= & |\delta(i-1)| \sup _{z \in E}|z| \leqslant p^{k_{i-1}}(|x|+d) \\
& <p^{k_{i}}(|x|-d) \leqslant|\delta(i)| \inf _{z \in E}|z|=\inf _{z \in E}|\delta(i) z| .
\end{aligned}
$$

3.5. Proposition. Let $\mathbf{G}$ be a connected F-group and $\mathbf{N}$ be its unipotent radical. Then there exists a layering $G=X_{0} \supset X_{1} \supset \cdots \supset X_{m}=Z_{G}(N)$ of closed normal subgroups of $G$ such that for each $x \in X_{j}-X_{j+1}$, there exists a relative neighborhood $V$ of $x$ in $X_{j}$ such that $V$ has countably many disjoint conjugates.

Proof. Let $N=N^{0} \supset N^{1} \supset \cdots \supset N^{m}=\{1\}$ be the central descending sequence of $N$. Then for each $j, N^{j}$ is a closed normal subgroup of $G$ and $N^{j} / N^{j+1}$ is topological isomorphic to $F^{r}$ for some natural number $r$. For each $j=0, \ldots, m$, define

$$
X_{j}=\left\{x \in G \mid[N, x] \subset N^{j}\right\} .
$$

Then we have that $G=X_{0} \supset X_{1} \supset \cdots \supset X_{m}=Z_{G}(N)$ and that $X_{j} \supset N^{j}, j=$ $0, \ldots, m$. As each $X_{j} / N^{j}$ is the centralizer of $N / N^{j}$ in $G / N^{j}$, so each $X_{j} / N^{j}$ is a closed normal subgroup of $G / N^{j}$ and hence each $X_{j}$ is a closed normal subgroup of $G$.

We show that the subgroups $X_{j}$ provide the desired layering of $G$. For $j=0, \ldots, m-1$, let $x \in X_{j}-X_{j+1}$; since $[N, x]$ is contained in $N^{j}$ but not in $N^{j+1}$, there is an element $u \in N$ such that $[u, x] \in N^{j}-N^{j+1}$. As the mapping $y \mapsto[u, y]$ from $X_{j}$ to $N^{j}$ is continuous, it follows that there exists a compact relative neighborhood $V$ of $x$ in $X_{j}$ such that $[u, y] \in N^{j}-N^{j+1}$ for all $y \in V$; since $X_{j+1}$ is closed, we may suppose further that $V \subset X_{j}-X_{j+1}$.

Let $\sim: G \rightarrow G / N^{j+1}$ be the natural projection and $\eta: X_{j}^{\sim} \rightarrow\left(N^{j}\right)^{\sim}$ be the continuous mapping defined by $\eta\left(y^{\sim}\right)=\left[u^{\sim}, y^{\sim}\right], y \in X_{j}$. Since $\left(N^{j}\right)^{\sim}$ is topological isomorphic to $F^{r}$ for some natural number $r$ and $\eta\left(x^{\sim}\right) \neq 1^{\sim}$, we have, by 3.4, an open set $E$ containing $\eta\left(x^{\sim}\right)$ in $\left(N^{j}\right)^{\sim}$ and a sequence of natural numbers $k_{1}<k_{2}<\cdots$ such that $E^{\delta(i)}$ are pairwise disjoint, where $\delta(i)=p^{k_{i}}$ if 
$F=\mathbf{R}$ or $\mathbf{C}$ and $\delta(i)=1 / p^{k_{i}}$ otherwise. Shrink $V$ if necessary, so that $\eta\left(V^{\sim}\right) \subset E$.

Let $W=\left(N^{j}\right)^{\sim} \cap V^{\sim} V^{\sim-1}$ and $W^{1 / 2}=\left\{w^{1 / 2} \mid w \in W\right\}$ (note here that $W$ is a symmetric neighborhood of $1^{\sim}$ in $\left.\left(N^{j}\right)^{\sim}\right)$. Then we have

$$
\text { for large } i\left(i \geqslant i_{0} \text { say), } \eta\left(V^{\sim}\right)^{\delta(i)} \cdot W^{1 / 2}\right. \text { pairwise disjoint. }
$$

Indeed, since $|1 /(2 \delta(i))| \rightarrow 0$ as $i \rightarrow \infty, W$ is compact and $\eta\left(V^{\sim}\right)$ is a compact set contained in the open set $E$, there exists an $i_{0}$ such that for all $i \geqslant i_{0}$, $\eta\left(V^{\sim}\right) \cdot W^{1 /(2 \delta(i))} \subset E$. Hence for all $i \geqslant i_{0}$,

$$
\eta\left(V^{\sim}\right)^{\delta(i)} \cdot W^{1 / 2}=\left(\eta\left(V^{\sim}\right) \cdot W^{1 /(2 \delta(i))}\right)^{\delta(i)} \subset E^{\delta(i)}
$$

are pairwise disjoint.

We claim that for $i \geqslant i_{0}, u^{\delta(i)} V u^{-\delta(i)}$ are pairwise disjoint. We observe first that every element in $N$ has a unique $i$ th root since $\exp _{\mid N}$ is a bijection. Now for any $y \in V$, since $[u, y] \notin N^{j+1}$, it is obvious that for any natural number $i$, both $[u, y]^{i}$ and $[u, y]^{1 / i}$ are not in $N^{j+1}$; in other words, both $\eta\left(y^{\sim}\right)^{i}$ and $\eta\left(y^{\sim}\right)^{1 / i}$ are distinct from $1^{\sim}$.

Next we prove that for all $y \in V, i=1,2, \ldots$, we have

$$
\begin{gathered}
u^{\sim 1 / i} y^{\sim} u^{\sim-1 / i}=\eta\left(y^{\sim}\right)^{1 / i} y^{\sim}, \\
u^{\sim i} y^{\sim} u^{\sim-i}=\eta\left(y^{\sim}\right)^{i} y^{\sim} .
\end{gathered}
$$

Consider any $u_{1}, u_{2} \in N$ and $y \in V,\left[u_{1}, y\right] \in N^{j}$ implies that $\left[u_{2},\left[u_{1}, y\right]\right] \in$ $N^{j+1}$. Hence $\left[u_{1}^{\sim}, y^{\sim}\right] u_{2}^{\tilde{z}}=u_{2}^{\tilde{2}}\left[u_{1}^{\tilde{1}}, y^{\sim}\right]$. Therefore for any $y \in V$ and $i, k=$ $1,2, \ldots$, we have

$$
\begin{aligned}
{\left[u^{\sim k / i}, y^{\sim}\right]\left[u^{\sim 1 / i}, y^{\sim}\right] } & =u^{\sim 1 / i}\left(u^{\sim k / i} y^{\sim} u^{\sim-k / i} y^{\sim-1}\right)\left(y^{\sim} u^{\sim-1 / i} y^{\sim-1}\right) \\
& =\left[u^{\sim(k+1) / i}, y^{\sim}\right] .
\end{aligned}
$$

Applying (3) $i-1$ times to the product $\left[u^{\sim 1 / i}, y^{\sim}\right]^{i}$, we have $\left[u^{\sim 1 / i}, y^{\sim}\right]^{i}=$ $\left[u^{\sim}, y^{\sim}\right]=\eta\left(y^{\sim}\right)$, or equivalently, $\left[u^{\sim 1 / i}, y^{\sim}\right]=\eta\left(y^{\sim}\right)^{1 / i}$. Hence (2) follows. Similarly applying (3) $i-1$ times to the product $\left[u^{\sim}, y^{\sim}\right]^{i}$, we obtain $\left(2^{\prime}\right)$.

Now suppose that there were $k>i \geqslant i_{0}$ such that $u^{\delta(i)} V u^{-\delta(i)} \cap$ $u^{\delta(k)} V u^{-\delta(k)} \neq \varnothing$. Then there would be $y_{1}, y_{2}$ in $V$ such that $u^{\delta(i)} y_{1} u^{-\delta(i)}=$ $u^{\delta(k)} y_{2} u^{-\delta(k)}$. Hence by (2) and (2'), we would have

$$
\eta\left(y_{1}^{\sim}\right)^{\delta(i)} y_{1}^{\sim}=\eta\left(y_{2}^{\sim}\right)^{\delta(k)} y_{2}^{\tilde{z}}
$$

or

$$
\eta\left(y_{1}^{\sim}\right)^{\delta(i)} \eta\left(y_{2}^{\sim}\right)^{-\delta(k)}=y_{2}^{\tilde{y}} y_{1}^{-1} \in\left(N^{j}\right)^{\sim} \cap V^{\sim} V^{\sim-1}=W .
$$

Thus there would be $w_{1}, w_{2} \in W^{1 / 2}$ such that

$$
\eta\left(y_{1}^{\sim}\right)^{\delta(i)} w_{1}=\eta\left(y_{2}^{\tilde{2}}\right)^{\delta(k)} w_{2} .
$$


This contradicts (1). Hence the claim is proved and the proof of 3.5 is complete.

3.6. Lemma. Let $\mathrm{T}$ be an F-split torus and $\psi_{1} \neq \psi_{2}$ be two characters of T. Then there exists a $t_{0} \in T$ such that the norm $\left|\psi_{1}\left(t_{0}\right) \psi_{2}\left(t_{0}\right)^{-1}\right| \neq 1$.

Proof. As mentioned in $\S 2$, we may assume that $\mathrm{T}=\mathrm{GL}(1)^{r}, T=$ $\mathrm{GL}(1, F)^{r}$ for some natural number $r$ and $\psi_{i}(t)=\Pi a_{j}^{m(i, j)}$ where $t=\left(a_{1}, \ldots, a_{r}\right)$ $\in \mathbf{T}, i=1,2$, and $m(i, j)$ are some suitable integers. Since $\psi_{1} \neq \psi_{2}$, there exists a $j, 1 \leqslant j \leqslant r$, such that $m(1, j) \neq m(2, j)$.

Let $t_{0}=\left(1, \ldots, 1, a_{j}, 1, \ldots, 1\right)$ with $a_{j} \in F^{\times}$such that $\left|a_{j}\right| \neq 1$. Then obviously $\psi_{1}\left(t_{0}\right) \psi_{2}\left(t_{0}\right)^{-1}=a_{j}^{m(1, j)-m(2, j)}$ has norm different from 1 .

3.7. Proposition. Let $\mathrm{T}$ be an F-split torus of $\mathrm{G}$. Then for any $x \in$ $G-Z_{G}(T)$, there exists a compact neighborhood $V$ of $x$ such that $V \subset G-Z_{G}(T)$ and $V$ has countably many disjoint conjugates.

Proof. Since $\mathrm{T}$ is a torus defined and split over $F$, there exists a basis $v_{1}, \ldots, v_{n}$ of $F^{n}$ such that $t\left(v_{i}\right)=\psi_{i}(t) v_{i}, t \in \mathrm{T}, i=1, \ldots, n$, where each $\psi_{i}$ is a character of $\mathrm{T}$. For each $x \in \mathrm{G}$, we have $x\left(v_{i}\right)=\sum_{j=1}^{n} x_{i j} v_{j}, i=1, \ldots, n$, where each $x_{i j}$ is a function defined over $F$ and $x_{i j \mid G}$ is continuous. Now

$$
\begin{aligned}
x \in Z_{G}(T)=Z_{G}(T) & \Longleftrightarrow(x t)_{i j}=(t x)_{i j}, \quad t \in T, 1 \leqslant i, j \leqslant n, \\
& \Longleftrightarrow x_{i j} \psi_{i}(t)=\psi_{j}(t) x_{i j} \\
& \Longleftrightarrow x_{i j}=0 \text { or } x_{i j} \neq 0 \text { and } \psi_{i}=\psi_{j} .
\end{aligned}
$$

Therefore $x \in G-Z_{G}(T) \Longleftrightarrow \exists i, j$ such that $x_{i j} \neq 0$ and $\psi_{i} \neq \psi_{j}$. So if we set $J=\left\{(i, j) \mid 1 \leqslant i, j \leqslant n, \psi_{i} \neq \psi_{j}\right\}$ and $U_{i j}=\left\{x \in G \mid x_{i j} \neq 0\right\}$, then it is obvious that each $U_{i j}$ is an open set in $G$ and that

$$
G-Z_{G}(T)=\bigcup\left\{U_{i j} \mid(i, j) \in J\right\} .
$$

Now let $x \in G-Z_{G}(T)$, then there exists $(i, j) \in J$ such that $x \in U_{i j}$. For this fixed $(i, j)$, let $V$ be a compact neighborhood of $x$ in $G$ such that $V \subset U_{i j}$. Let $c, c^{\prime}$ be such that

$$
0<c=\min _{x \in V}\left|x_{i j}\right| \leqslant \max _{x \in V}\left|x_{i j}\right|=c^{\prime} .
$$

Since $\psi_{i} \neq \psi_{j}$, it follows from 3.5 that there exists a $t_{0} \in T$ such that $\left|\psi_{i}\left(t_{0}\right) \psi_{j}\left(t_{0}\right)^{-1}\right|=a \neq 1$. Also for any $t \in \mathbf{T}$ and any integer $k$, we have

$$
\left(t^{k} x t^{-k}\right)_{i j}=\psi_{i}(t)^{k} x_{i j} \psi_{j}(t)^{-k}=\left(\psi_{i}(t) \psi_{j}(t)^{-1}\right)^{k} x_{i j}
$$

and in particular, we have $\left|\left(t_{0}^{k} x t_{0}^{-k}\right)_{i j}\right|=a^{k}\left|x_{i j}\right|$. So if we pick an integer $k$ such that $a^{k} c>c^{\prime}$, then the sequence of conjugates $V_{m}=t_{0}^{m k} V t_{0}^{-m k}$ are pairwise disjoint since 


$$
\min _{x \in V_{m+1}}\left|x_{i j}\right|=a^{(m+1) k} c>a^{m k} c^{\prime}=\max _{x \in V_{m}}\left|x_{i j}\right| .
$$

\subsection{Proposition. Let $\mathbf{G}$ be a connected F-group. Then $G \supset \cap Z_{G}(T)$} $(\mathrm{T} \in T)$ is a 1-layering of $G$.

Proof. We recall that $\bigcap Z_{G}(T)$ ( $\left.\mathrm{T} \in T\right)$ is a closed normal subgroup of $G$. Now for any $x \in G-\cap Z_{G}(T)$, there exists a $T \in T$ such that $x \in G-Z_{G}(T)$. Hence it is easy to see that the proposition follows from 3.7.

It is now clear that 3.2 follows from $3.3,3.5$ and 3.8.

REMARK. In the proof of 3.5 , if we define

$$
X_{j}=\left\{x \in G \mid[N, \alpha(x)] \subset N^{j}, \alpha \in B\right\}
$$

where $B$ denotes the group of all $F$-rational a.b.d. of $\mathbf{G}$, then it can be shown that $G=X_{0} \supset \cdots \supset X_{m}=Z_{G}(N)$ is a layering of $G$ with the additional property that the $X_{j}$ 's are $B$-invariant. Also the 1-layering $G \supset \bigcap Z_{G}(T)(T \in T)$ is B-invariant. Hence we can obtain a $B$-invariant layering of $G$ terminating at $B(G)$.

4. Applications. We use the notations of the previous sections.

First let us recall some facts in measure theory: Let $\mathbf{G}$ be a connected $F$ group and $G$ its $F$-rational points. Since $F$ is a countable union of compacts, it follows that $G$ and the conjugacy class $\Gamma_{x}$ of $x \in G$ have the same property. Let $C_{x}$ be the centralizer of $x$ in $G$. As $G / C_{x}$ and $\Gamma_{x}$ are standard Borel spaces, the canonical bijection $\eta: G / C_{x} \rightarrow \Gamma_{x}$ is a continuous Borel isomorphism. Furthermore, $\eta$ is equivariant with respect to the left translation of $G$ on $G / C_{x}$ and the action of $I(G)$ on $\Gamma_{x}$. Thus (finite) left invariant measure on $G / C_{x}$ can be transferred to (finite) $I(G)$-invariant measure on $\Gamma_{x}$ and vice versa. The latter measure can be regarded as central measure on $G$ supported on $\Gamma_{\boldsymbol{x}}$.

Proof of 4.4. Since $G / C_{x}$ admits a finite invariant measure $\mu$, we may regard $\mu$ as a finite central measure on $G$ supported on $\Gamma_{x}$. Therefore by 3.1, we have $x \in \Gamma_{x} \subset B(G)$. As observed in the remark after the proof of 2.1, we have $\Gamma_{x}$ compact. Now by standard Baire category arguments it is easy to see that $G / C_{x}$ is compact if and only if $\Gamma_{x}$ is compact (see for example [5, Lemma 1]).

Proof of 4.1. Let $x \in Z_{G}(H)$. Then $H \subset C_{x}$ and the continuous surjection $\phi: G / H \rightarrow G / C_{x}$ induces a finite invariant measure $\mu^{\prime}: \mu^{\prime}(E)=\mu\left(\phi^{-1}(E)\right)$ on $G / C_{\boldsymbol{x}}$. Hence as in the proof of 4.4 we have $x \in \Gamma_{\boldsymbol{x}} \subset B(G)$ and so the proof of 4.1 is complete.

Since $B(G)$ is an $[F D]^{-}$group (Corollary 2.7) and since every closed subgroup of an $[F D]^{-}$group is again an $[F D]^{-}$group, 4.2 follows immediately from 4.1. Also it is clear that 4.3 is an immediate consequence of 4.1 , since $Z(G) \subset$ $Z_{G}(H) \subset B(G)=Z(G)$. 


\section{REFERENCES}

1. A. Borel, Density properties for certain subgroups of semisimple groups without compact components, Ann. of Math. (2) 72 (1960), 179-188. MR 23 \#A964.

2. Linear algebraic groups, Benjamin, New York, 1969. MR 40 \#4273.

3. A. Borel and J. Tits, Groupes réductifs, Inst. Hautes Études Sci. Publ. Math. No. 27 (1965), 55-150. MR 34 \#7527.

4. F. Greenleaf, M. Moskowitz and L. P. Rothschild, Unbounded conjugacy classes and location of central measures, Acta Math. (to appear).

Math. (to appear).

5. Compactness of certain homogeneous spaces of finite volume, Amer. J.

6. S. Grosser and M. Moskowitz, Compactness conditions in topological groups, J. Reine Angew Math. 246 (1971), 1-40. MR 44 \#1766.

7. Harish-Chandra, Harmonic analysis on reductive p-adic groups, Lecture Notes in Math., vol. 162, Springer-Verlag, Berlin and New York, 1970.

8. G. Hochschild, The structure of Lie groups, Holden-Day, San Francisco, Calif., 1965. MR 34 \#7696.

9. G. D. Mostow, Homogeneous spaces with finite invariant measure, Ann. of Math. (2) 75 (1962), 17-37. MR 26 \#2546.

10. D. L. Ragozin and L. P. Rothschild, Central measures on semisimple Lie groups have essentially compact support, Proc. Amer. Math. Soc. 32 (1972), 585-589. MR 45 \#466.

11. K.-Y. Sit, On centralizers of generalized uniform subgroups of locally compact groups, Trans. Amer. Math. Soc. 201 (1975), 133-146.

12. J. Tits, Automorphismes à déplacement borné des groupes de Lie, Topology 3 (1964), suppl. 1, 97-107. MR 28 \#2170.

13. T. Tamagawa, On discrete subgroups of p-adic algebraic groups, Arithmetical Algebraic Geometry (Proc. Conf. Purdue Univ., 1963), Harper \& Row, New York, 1965, pp. 11-17. MR 33 \#4060.

DEPARTMENT OF MATHEMATICS, YORK COLLEGE (CUNY), JAMAICA, NEW YORK 11432 